\title{
Initial-boundary value problem for the two-component nonlinear Schrödinger equation on the half-line
}

Jian Xu

To cite this article: Jian Xu (2016) Initial-boundary value problem for the twocomponent nonlinear Schrödinger equation on the half-line, Journal of Nonlinear Mathematical Physics 23:2, 167-189, DOI:

https://doi.org/10.1080/14029251.2016.1161259

To link to this article: https://doi.org/10.1080/14029251.2016.1161259

Published online: 04 January 2021 


\title{
Initial-boundary value problem for the two-component nonlinear Schrödinger equation on
} the half-line

\author{
Jian Xu \\ College of Science, University of Shanghai for Science and Technology, NO.334 Jungong Road \\ Shanghai, 200093, People's Republic of China \\ jianxu@usst.edu.cn
}

Received 16 October 2015

Accepted 19 January 2016

\begin{abstract}
We present a $3 \times 3$ Riemann-Hilbert problem formalism for the initial-boundary value problem of the twocomponent nonlinear Schrödinger (2-NLS) equation on the half-line. And we also get the Dirichlet to Neuemann map through analysising the global relation in this paper.

Keywords: Riemann-Hilbert problem; Initial-boundary value problem; two-component nonlinear Schrödinger equation.
\end{abstract}

2000 Mathematics Subject Classification: 37K40, 35Q53

\section{Introduction}

Integrable PDEs can be written as the compatibility condition of two linear eigenvalue equations, which are called Lax pairs [9]. In 1967, Gardner, Greene, Kruskal, Miura [7] used the Inverse Scattering Transform (IST) formalism to analyze the initial value problem for KdV equation. From then on, the IST method became a powerful tool to analyze the other integrable PDEs, such as NLS equation, Sine-Gordon equation and so on. Until the 1990s the IST methodology was pursued almost entirely for pure initial value problems. However, in many situations, we need to consider an initial-boundary value (IBV) problem instead of a pure initial value problem.

In 1997, Fokas announced a new unified approach for the analysis of IBV problems for linear and nonlinear integrable PDEs [2,3](see also [4]). The Fokas method provides a generalization of the IST formalism from initial value to IBV problems. As the IST on the line, the unified method yields an expression for the solution of an IBV problem in terms of the solution of a RiemannHilbert problem. But there has a big difference between the unified method and IST method. The IST method just need analyze the x-part of Lax pairs to obtain the scattering data, the other t-part of 
Lax pairs is used to determine the time-dependent of the scattering data. But the unified method need analyze simultaneously the x-part and t-part of Lax pairs. This difference leads to some difficulties in analyzing the IBV problem. Usually, the derivative order involved in the t-part of Lax pairs is less than the original integrable PDEs one. Hence, we need know all these boundary data to the spectral analysis. However, it needs "more" boundary data than a well-posed IBV problem. We need determine these "more" boundary data. This can be done by analyzing the global relation, which expresses the initial value data and all boundary data are not independent.

The unified method was usually used to analyze the IBV problem for integrable PDES with $2 \times 2$ Lax pairs [1,5] (see also a series of paper $[6,12,13]$ ). But many important integrable PDEs involve $3 \times 3$ Lax pairs, such as Boussnissq equation, DP equation, manakov systems and so on. It naturally leads to extend unified method from $2 \times 2$ Lax pairs to $3 \times 3$ Lax pairs. In 2012, Lenells developed an extended methodology for analyzing IBV problems for integrable evolution equations with Lax pairs involving $3 \times 3$ matrices [10], Degasperis-Procesi equation [11]. And some other $3 \times 3$ integrable equations, such as Sasa-Satsuma equation [15], the three-wave equation [16] are considered by the authors.

In this paper, we will consider IBV problem for the manakov systems. It is well known that the nonlinear Schrödinger(NLS) equation

$$
i q_{t}+q_{x x}+2 \sigma|q|^{2} q=0, \quad \sigma= \pm 1
$$

describes slowly varying wave envelopes in dispersive media and arises in various physical systems such as water waves, plasma physics, solid-state physics and nonlinear optics. One of the most successful among them is the description of optical solitons in fibers. But, many complex systems, such as nonlinear optics, etal, involves more than one-component. It is nature to extend the studies to the two-component case, see [14].

The two-component nonlinear Schrödinger equation or Manokov equation is

$$
\left\{\begin{array}{l}
i q_{1 t}+q_{1 x x}-2 \sigma\left(\left|q_{1}\right|^{2}+\left|q_{2}\right|^{2}\right) q_{1}=0, \\
i q_{2 t}+q_{2 x x}-2 \sigma\left(\left|q_{1}\right|^{2}+\left|q_{2}\right|^{2}\right) q_{2}=0 .
\end{array} \quad \sigma= \pm 1 .\right.
$$

Here, $\sigma=1$ means defocusing case and $\sigma=-1$ means focusing case. In this paper, we consider the defocusing case $\mathrm{c}^{\mathrm{a}}$ on the half-line $x \geq 0$. That is to say, we consider the following initial-boundary

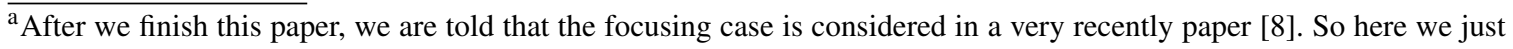
consider the defocusing case. And we compare this paper with the paper [8], the Lax pairs of equation (1.2) are chosen different form and we find that although we all use the same unified method, but we use block matrix to analyze the global relation, as we did in [15]. And in the results of Dirichlet to Neumann map, we also consider the possible finite simple poles, which wasn't considered in [8].
} 
value problem of the 2-NLS equation,

$$
\begin{array}{ll}
q_{10}(x)=q_{1}(x, t=0), & q_{20}(x)=q_{2}(x, t=0), \\
g_{01}(t)=q_{1}(x=0, t), & g_{02}(t)=q_{2}(x=0, t), \\
g_{11}(t)=q_{1 x}(x=0, t), & g_{12}(t)=q_{2 x}(x=0, t) .
\end{array}
$$

where $q_{10}(x)$ and $q_{20}(x)$ lie in Schwartz space.

In fact, the most important motivation for us to consider this IBV problem for 2-component NLS equation or manakov systems is that we try to extend the IBV problem on the half-line to the IBV problem on the finite interval for integrable PDEs with $3 \times 3$ Lax pairs. And we indeed do this extension in the paper [17], where we consider both the defocusing and focusing case. And the results about the Dirichlet to Neumann map obtained in this paper can be viewed as the results of the length of the finite interval goes to infity, in [17].

Organization of the paper: In section 2 we perform the spectral analysis of the associated Lax pair. And we formulate the main Riemann-Hilbert problem in section 3. We also get the map between the Dirichlet and the Neumann boundary problem through analysising the global relation in section 4 .

\section{Spectral analysis}

The 2-NLS equation admits a $3 \times 3$ Lax pair,

$$
\begin{gathered}
\Psi_{x}=U \Psi, \quad \Psi=\left(\begin{array}{l}
\Psi_{1} \\
\Psi_{2} \\
\Psi_{3}
\end{array}\right) . \\
\Psi_{t}=V \Psi .
\end{gathered}
$$

where

$$
U=i k \Lambda+V_{1} .
$$

and

$$
V=2 i k^{2} \Lambda+V_{2}
$$

here

$$
\Lambda=\left(\begin{array}{ccc}
-1 & 0 & 0 \\
0 & 1 & 0 \\
0 & 0 & 1
\end{array}\right), V_{1}=\left(\begin{array}{ccc}
0 & q_{1} & q_{2} \\
\bar{q}_{1} & 0 & 0 \\
\bar{q}_{2} & 0 & 0
\end{array}\right), V_{2}=k V_{2}^{(1)}+V_{2}^{(0)}
$$


where

$$
V_{2}^{(1)}=2 V_{1}, \quad V_{2}^{(0)}=i \Lambda\left(V_{1}^{2}-V_{1 x}\right)
$$

\subsection{The closed one-form}

Suppose that $u(x, t)$ is sufficiently smooth function of $(x, t)$ in the half-line domain $\Omega=\{0<x<$ $\infty, 0<t<T\}$ which decay as $x \rightarrow \infty$. Introducing a new eigenfunction $\mu(x, t, k)$ by

$$
\Psi=\mu e^{i \Lambda k x+2 i \Lambda k^{2} t}
$$

then we find the Lax pair equations

$$
\left\{\begin{array}{l}
\mu_{x}-[i k \Lambda, \mu]=V_{1} \mu, \\
\mu_{t}-\left[2 i k^{2} \Lambda, \mu\right]=V_{2} \mu
\end{array}\right.
$$

Letting $\hat{A}$ denotes the operators which acts on a $3 \times 3$ matrix $X$ by $\hat{A} X=[A, X]$, then the equations in (2.7) can be written in differential form as

$$
d\left(e^{-\left(i k x+2 i k^{2} t\right) \hat{\Lambda}} \mu\right)=W
$$

where $W(x, t, k)$ is the closed one-form defined by

$$
W=e^{-\left(i k x+2 i k^{2} t\right) \hat{\Lambda}}\left(V_{1} d x+V_{2} d t\right) \mu .
$$

\subsection{The $\mu_{j}$ 's}

We define three eigenfunctions $\left\{\mu_{j}\right\}_{1}^{3}$ of (2.7) by the Volterra integral equations

$$
\mu_{j}(x, t, k)=\mathbb{I}+\int_{\gamma_{j}} e^{\left(i k x+2 i k^{2} t\right) \hat{\Lambda}} W_{j}\left(x^{\prime}, t^{\prime}, k\right) . \quad j=1,2,3 .
$$

where $W_{j}$ is given by (2.9) with $\mu$ replaced with $\mu_{j}$, and the contours $\left\{\gamma_{j}\right\}_{1}^{3}$ are showed in Figure 1. The first, second and third column of the matrix equation (2.10) involves the exponentials
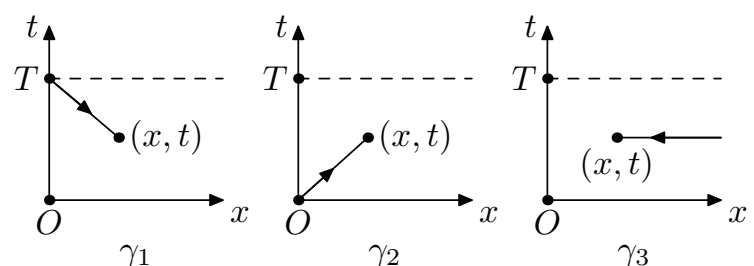

Fig. 1. The three contours $\gamma_{1}, \gamma_{2}$ and $\gamma_{3}$ in the $(x, t)$-domain.

Co-published by Atlantis Press and Taylor \& Francis

Copyright: the authors 


$$
\begin{aligned}
& {\left[\mu_{j}\right]_{1}: e^{2 i k\left(x-x^{\prime}\right)+4 i k^{2}\left(t-t^{\prime}\right)}, e^{2 i k\left(x-x^{\prime}\right)+4 i k^{2}\left(t-t^{\prime}\right)}} \\
& {\left[\mu_{j}\right]_{2}: e^{-2 i k\left(x-x^{\prime}\right)-4 i k^{2}\left(t-t^{\prime}\right)}} \\
& {\left[\mu_{j}\right]_{3}: e^{-2 i k\left(x-x^{\prime}\right)-4 i k^{2}\left(t-t^{\prime}\right)}}
\end{aligned}
$$

And we have the following inequalities on the contours:

$$
\begin{aligned}
& \gamma_{1}: x-x^{\prime} \geq 0, t-t^{\prime} \leq 0 \\
& \gamma_{2}: x-x^{\prime} \geq 0, t-t^{\prime} \geq 0 \\
& \gamma_{3}: x-x^{\prime} \leq 0
\end{aligned}
$$

So, these inequalities imply that the functions $\left\{\mu_{j}\right\}_{1}^{3}$ are bounded and analytic for $k \in \mathbb{C}$ such that $k$ belongs to

$$
\begin{aligned}
& \mu_{1}:\left(D_{2}, D_{3}, D_{3}\right), \\
& \mu_{2}:\left(D_{1}, D_{4}, D_{4}\right), \\
& \mu_{3}:\left(D_{3} \cup D_{4}, D_{1} \cup D_{2}, D_{1} \cup D_{2}\right) .
\end{aligned}
$$

where $\left\{D_{n}\right\}_{1}^{4}$ denote four open, pairwisely disjoint subsets of the complex $k$-sphere showed in Figure 2. And the sets $\left\{D_{n}\right\}_{1}^{4}$ has the following properties:

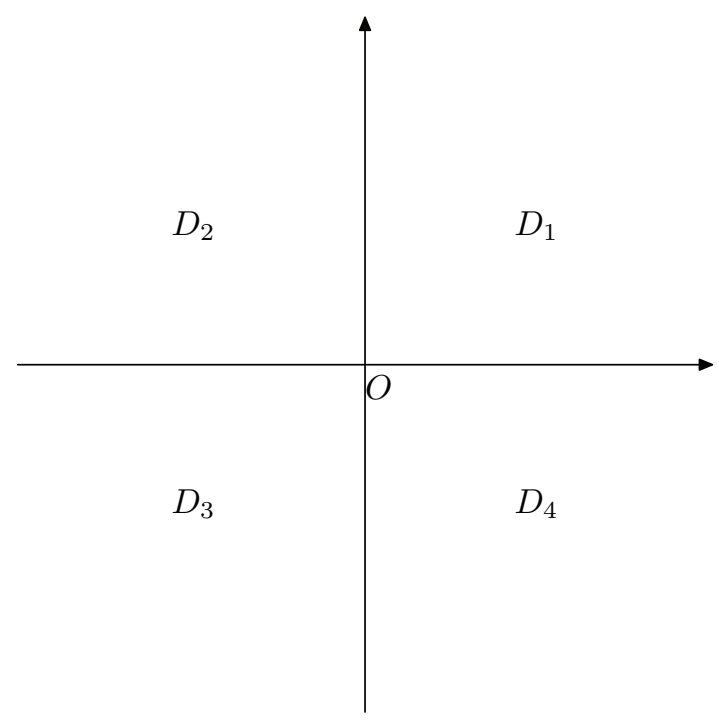

Fig. 2. The sets $D_{n}, n=1, \ldots, 4$, which decompose the complex $k$-plane.

$$
\begin{aligned}
& D_{1}=\left\{k \in \mathbb{C} \mid \operatorname{Re} l_{1}>\operatorname{Re} l_{2}=\operatorname{Re} l_{3}, \operatorname{Re} z_{1}>\operatorname{Re} z_{2}=\operatorname{Re} z_{3}\right\}, \\
& D_{2}=\left\{k \in \mathbb{C} \mid \operatorname{Re} l_{1}>\operatorname{Re} l_{2}=\operatorname{Re} l_{3}, \operatorname{Re} z_{1}<\operatorname{Re} z_{2}=\operatorname{Re} z_{3}\right\}, \\
& D_{3}=\left\{k \in \mathbb{C} \mid \operatorname{Re} l_{1}<\operatorname{Re} l_{2}=\operatorname{Re} l_{3}, \operatorname{Re} z_{1}>\operatorname{Re} z_{2}=\operatorname{Re} z_{3}\right\}, \\
& D_{4}=\left\{k \in \mathbb{C} \mid \operatorname{Re} l_{1}<\operatorname{Re} l_{2}=\operatorname{Re} l_{3}, \operatorname{Re} z_{1}<\operatorname{Re} z_{2}=\operatorname{Re} z_{3}\right\},
\end{aligned}
$$


where $l_{i}(k)$ and $z_{i}(k)$ are the diagonal entries of matrices $i k \Lambda$ and $2 i k^{2} \Lambda$, respectively.

In fact, for $x=0, \mu_{1}(0, t, k)$ has enlarged domain of boundedness: $\left(D_{2} \cup D_{4}, D_{1} \cup D_{3}, D_{1} \cup D_{3}\right)$, and $\mu_{2}(0, t, k)$ has enlarged domain of boundedness: $\left(D_{1} \cup D_{3}, D_{2} \cup D_{4}, D_{2} \cup D_{4}\right)$.

\subsection{The $M_{n}$ 's}

For each $n=1, \ldots, 4$, define a solution $M_{n}(x, t, k)$ of (2.7) by the following system of integral equations:

$$
\left(M_{n}\right)_{i j}(x, t, k)=\delta_{i j}+\int_{\gamma_{i j}^{n}}\left(e^{\left(i k x+2 i k^{2} t\right) \hat{\Lambda}} W_{n}\left(x^{\prime}, t^{\prime}, k\right)\right)_{i j}, \quad k \in D_{n}, \quad i, j=1,2,3 .
$$

where $W_{n}$ is given by (2.9) with $\mu$ replaced with $M_{n}$, and the contours $\gamma_{i j}^{n}, n=1, \ldots, 4, i, j=1,2,3$ are defined by

$$
\gamma_{i j}^{n}=\left\{\begin{array}{l}
\gamma_{1} \text { if } \operatorname{Re}_{i}(k)<\operatorname{Re}_{j}(k) \text { and } \operatorname{Re}_{i}(k) \geq \operatorname{Re} z_{j}(k), \\
\gamma_{2} \text { if } \operatorname{Re}_{i}(k)<\operatorname{Re} l_{j}(k) \text { and } \operatorname{Re} z_{i}(k)<\operatorname{Re} z_{j}(k), \quad \text { for } \quad k \in D_{n} . \\
\gamma_{3} \text { if } \operatorname{Re}_{i}(k) \geq \operatorname{Re}_{j}(k)
\end{array}\right.
$$

The following proposition ascertains that the $M_{n}$ 's defined in this way have the properties required for the formulation of a Riemann-Hilbert problem.

Proposition 2.1. For each $n=1, \ldots, 4$, the function $M_{n}(x, t, k)$ is well-defined by equation (2.14) for $k \in \bar{D}_{n}$ and $(x, t) \in \Omega$. Moreover, $M_{n}$ admits a bounded and contious extension to $\bar{D}_{n}$ and

$$
M_{n}(x, t, k)=\mathbb{I}+O\left(\frac{1}{k}\right), \quad k \rightarrow \infty, \quad k \in D_{n} .
$$

Proof. Substituting the expansion

$$
M=M_{0}+\frac{M^{(1)}}{k}+\frac{M^{(2)}}{k^{2}}+\cdots, \quad k \rightarrow \infty .
$$

into the Lax pair (2.7) and comparing the terms of the same order of $k$ yield the equation (2.16).

Remark 2.1. Of course, for any fixed point $(x, t), M_{n}$ is bounded and analytic as a function of $k \in D_{n}$ away from a possible discrete set of singularities $\left\{k_{j}\right\}$ at which the Fredholm determinant vanishes. The bounedness and analyticity properties are established in appendix B in [10].

\subsection{The jump matrices}

We define spectral functions $S_{n}(k), n=1, \ldots, 4$, and

$$
S_{n}(k)=M_{n}(0,0, k), \quad k \in D_{n}, \quad n=1, \ldots, 4 .
$$


Let $M$ denote the sectionally analytic function on the Riemann $k$-sphere which equals $M_{n}$ for $k \in D_{n}$. Then $M$ satisfies the jump conditions

$$
M_{n}=M_{m} J_{m, n}, \quad k \in \bar{D}_{n} \cap \bar{D}_{m}, \quad n, m=1, \ldots, 4, \quad n \neq m,
$$

where the jump matrices $J_{m, n}(x, t, k)$ are defined by

$$
J_{m, n}=e^{\left(i k x+2 i k^{2} t\right) \hat{\Lambda}}\left(S_{m}^{-1} S_{n}\right)
$$

According to the definition of the $\gamma^{n}$, we find that

$$
\begin{aligned}
\gamma^{1} & =\left(\begin{array}{lll}
\gamma_{3} & \gamma_{3} & \gamma_{3} \\
\gamma_{2} & \gamma_{3} & \gamma_{3} \\
\gamma_{2} & \gamma_{3} & \gamma_{3}
\end{array}\right) \gamma^{2}=\left(\begin{array}{lll}
\gamma_{3} & \gamma_{3} & \gamma_{3} \\
\gamma_{1} & \gamma_{3} & \gamma_{3} \\
\gamma_{1} & \gamma_{3} & \gamma_{3}
\end{array}\right) \\
\gamma^{3} & =\left(\begin{array}{lll}
\gamma_{3} & \gamma_{1} & \gamma_{1} \\
\gamma_{3} & \gamma_{3} & \gamma_{3} \\
\gamma_{3} & \gamma_{3} & \gamma_{3}
\end{array}\right) \gamma^{4}=\left(\begin{array}{lll}
\gamma_{3} & \gamma_{2} & \gamma_{2} \\
\gamma_{3} & \gamma_{3} & \gamma_{3} \\
\gamma_{3} & \gamma_{3} & \gamma_{3}
\end{array}\right) .
\end{aligned}
$$

\subsection{The adjugated eigenfunctions}

As the expressions of $S_{n}(k)$ will involve the adjugate matrix of $\{s(k), S(k)\}$ defined in the next subsection. We will also need the analyticity and boundedness properties of the minors of the matrices $\left\{\mu_{j}(x, t, k)\right\}_{1}^{3}$. We recall that the adjugate matrix $X^{A}$ of a $3 \times 3$ matrix $X$ is defined by

$$
X^{A}=\left(\begin{array}{ccc}
m_{11}(X) & -m_{12}(X) & m_{13}(X) \\
-m_{21}(X) & m_{22}(X) & -m_{23}(X) \\
m_{31}(X) & -m_{32}(X) & m_{33}(X)
\end{array}\right),
$$

where $m_{i j}(X)$ denote the $(i j)$ th minor of $X$.

It follows from (2.7) that the adjugated eigenfunction $\mu^{A}$ satisfies the Lax pair

$$
\left\{\begin{array}{l}
\mu_{x}^{A}+\left[i k \Lambda, \mu^{A}\right]=-V_{1}^{T} \mu^{A}, \\
\mu_{t}^{A}+\left[2 i k^{2} \Lambda, \mu^{A}\right]=-V_{2}^{T} \mu^{A} .
\end{array}\right.
$$

where $V^{T}$ denote the transform of a matrix $V$. Thus, the eigenfunctions $\left\{\mu_{j}^{A}\right\}_{1}^{3}$ are solutions of the integral equations

$$
\mu_{j}^{A}(x, t, k)=\mathbb{I}-\int_{\gamma_{j}} e^{-i k\left(x-x^{\prime}\right)-2 i k^{2}\left(t-t^{\prime}\right) \hat{\Lambda}}\left(V_{1}^{T} d x+V_{2}^{T}\right) \mu^{A}, \quad j=1,2,3 .
$$

Then we can get the following analyticity and boundedness properties:

$$
\begin{aligned}
\mu_{1}^{A} & :\left(D_{3}, D_{2}, D_{2}\right), \\
\mu_{2}^{A} & :\left(D_{4}, D_{1}, D_{1}\right), \\
\mu_{3}^{A} & :\left(D_{1} \cup D_{2}, D_{3} \cup D_{4}, D_{3} \cup D_{4}\right) .
\end{aligned}
$$


In fact, for $x=0, \mu_{1}^{A}(0, t, k)$ has enlarged domain of boundedness: $\left(D_{1} \cup D_{3}, D_{2} \cup D_{4}, D_{2} \cup D_{4}\right)$, and $\mu_{2}^{A}(0, t, k)$ has enlarged domain of boundedness: $\left(D_{2} \cup D_{4}, D_{1} \cup D_{3}, D_{1} \cup D_{3}\right)$.

\subsection{The $J_{m, n}$ 's computation}

Let us define the $3 \times 3-$ matrix value spectral functions $s(k)$ and $S(k)$ by

$$
\begin{aligned}
& \mu_{3}(x, t, k)=\mu_{2}(x, t, k) e^{\left(i k x+2 i k^{2} t\right) \hat{\Lambda}} s(k), \\
& \mu_{1}(x, t, k)=\mu_{2}(x, t, k) e^{\left(i k x+2 i k^{2} t\right) \hat{\Lambda}} S(k),
\end{aligned}
$$

Thus,

$$
s(k)=\mu_{3}(0,0, k), \quad S(k)=\mu_{1}(0,0, k) .
$$

And we deduce from the properties of $\mu_{j}$ and $\mu_{j}^{A}$ that $s(k)$ and $S(k)$ have the following boundedness properties:

$$
\begin{array}{ll}
s(k): & \left(D_{3} \cup D_{4}, D_{1} \cup D_{2}, D_{1} \cup D_{2}\right), \\
S(k): & \left(D_{2} \cup D_{4}, D_{1} \cup D_{3}, D_{1} \cup D_{3}\right), \\
s^{A}(k): & \left(D_{1} \cup D_{2}, D_{3} \cup D_{4}, D_{3} \cup D_{4}\right), \\
S^{A}(k): & \left(D_{1} \cup D_{3}, D_{2} \cup D_{4}, D_{2} \cup D_{4}\right) .
\end{array}
$$

Moreover,

$$
M_{n}(x, t, k)=\mu_{2}(x, t, k) e^{\left(i k x+2 i k^{2} t\right) \hat{\Lambda}} S_{n}(k), \quad k \in D_{n} .
$$

Proposition 2.2. The $S_{n}$ can be expressed in terms of the entries of $s(k)$ and $S(k)$ as follows:

$$
\begin{aligned}
& S_{1}=\left(\begin{array}{ccc}
\frac{1}{m_{11}(s)} & s_{12} & s_{13} \\
0 & s_{22} & s_{23} \\
0 & s_{32} & s_{33}
\end{array}\right), S_{2}=\left(\begin{array}{c}
\frac{S_{11}}{\left(S^{T} s^{A}\right)_{11}} s_{12} s_{13} \\
\frac{S_{21}}{\left(S^{T} s^{A}\right)_{11}} s_{22} s_{23} \\
\frac{S_{31}}{\left(S^{T} s^{A}\right)_{11}} s_{32} s_{33}
\end{array}\right)
\end{aligned}
$$

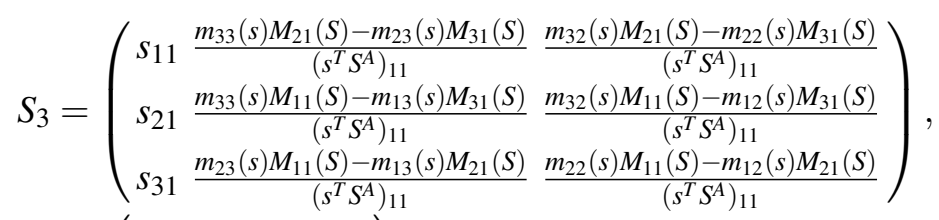

$$
\begin{aligned}
& S_{4}=\left(\begin{array}{ccc}
s_{11} & 0 & 0 \\
s_{21} & \frac{m_{33}(s)}{s_{11}} & \frac{m_{32}(s)}{s_{11}} \\
s_{31} & \frac{m_{23}(s)}{s_{11}} & \frac{m_{22}(s)}{s_{11}}
\end{array}\right) \text {. }
\end{aligned}
$$


Proof. Let $\gamma_{3}^{X_{0}}$ denote the contour $\left(X_{0}, 0\right) \rightarrow(x, t)$ in the $(x, t)$-plane, here $X_{0}>0$ is a constant. We introduce $\mu_{3}\left(x, t, k ; X_{0}\right)$ as the solution of (2.10) with $j=3$ and with the contour $\gamma_{3}$ replaced by $\gamma_{3}^{X_{0}}$. Similarly, we define $M_{n}\left(x, t, k ; X_{0}\right)$ as the solution of (2.14) with $\gamma_{3}$ replaced by $\gamma_{3}^{X_{0}}$. We will first derive expression for $S_{n}\left(k ; X_{0}\right)=M_{n}\left(0,0, k ; X_{0}\right)$ in terms of $S(k)$ and $s\left(k ; X_{0}\right)=\mu_{3}\left(0,0, k ; X_{0}\right)$. Then (2.27) will follow by taking the limit $X_{0} \rightarrow \infty$.

First, We have the following relations:

$$
\left\{\begin{array}{l}
M_{n}\left(x, t, k ; X_{0}\right)=\mu_{1}(x, t, k) e^{\left(i k x+2 i k^{2} t\right) \hat{\Lambda}} R_{n}\left(k ; X_{0}\right), \\
M_{n}\left(x, t, k ; X_{0}\right)=\mu_{2}(x, t, k) e^{\left(i k x+2 i k^{2} t\right) \hat{\Lambda}} S_{n}\left(k ; X_{0}\right), \\
M_{n}\left(x, t, k ; X_{0}\right)=\mu_{3}(x, t, k) e^{\left(i k x+2 i k^{2} t\right) \hat{\Lambda}} T_{n}\left(k ; X_{0}\right) .
\end{array}\right.
$$

Then we get $R_{n}\left(k ; X_{0}\right)$ and $T_{n}\left(k ; X_{0}\right)$ are fedined as follows:

$$
\begin{gathered}
R_{n}\left(k ; X_{0}\right)=e^{-2 i k^{2} T \hat{\Lambda}} M_{n}\left(0, T, k ; X_{0}\right), \\
T_{n}\left(k ; X_{0}\right)=e^{-i k x \hat{\Lambda}} M_{n}\left(X_{0}, 0, k ; X_{0}\right) .
\end{gathered}
$$

The relations (2.28) imply that

$$
s\left(k ; X_{0}\right)=S_{n}\left(k ; X_{0}\right) T_{n}^{-1}\left(k ; X_{0}\right), \quad S(k)=S_{n}\left(k ; X_{0}\right) R_{n}^{-1}\left(k ; X_{0}\right) .
$$

These equations constitute a matrix factorization problem which, given $\{s, S\}$ can be solved for the $\left\{R_{n}, S_{n}, T_{n}\right\}$. Indeed, the integral equations (2.14) together with the definitions of $\left\{R_{n}, S_{n}, T_{n}\right\}$ imply that

$$
\left\{\begin{array}{l}
\left(R_{n}\left(k ; X_{0}\right)\right)_{i j}=0 \text { if } \gamma_{i j}^{n}=\gamma_{1} \\
\left(S_{n}\left(k ; X_{0}\right)\right)_{i j}=0 \text { if } \gamma_{i j}^{n}=\gamma_{2} \\
\left(T_{n}\left(k ; X_{0}\right)\right)_{i j}=\delta_{i j} \text { if } \gamma_{i j}^{n}=\gamma_{3}
\end{array}\right.
$$

It follows that (2.30) are 18 scalar equations for 18 unknowns. By computing the explicit solution of this algebraic system, we find that $\left\{S_{n}\left(k ; X_{0}\right)\right\}_{1}^{4}$ are given by the equation obtained from (2.27) by replacing $\left\{S_{n}(k), s(k)\right\}$ with $\left\{S_{n}\left(k ; X_{0}\right), s\left(k ; X_{0}\right)\right\}$. taking $X_{0} \rightarrow \infty$ in this equation, we arrive at (2.27).

\subsection{The residue conditions}

Since $\mu_{2}$ is an entire function, it follows from (2.26) that $\mathrm{M}$ can only have sigularities at the points where the $S_{n}^{\prime} s$ have singularities. We denote the possible zeros by $\left\{k_{j}\right\}_{1}^{N}$ and assume they satisfy the following assumption. 
Assumption 2.1. We assume that

- $m_{11}(s)(k)$ has $n_{0}$ possible simple zeros in $D_{1}$ denoted by $\left\{k_{j}\right\}_{1}^{n_{0}}$;

- $\left(S^{T} s^{A}\right)_{11}(k)$ has $n_{1}-n_{0}$ possible simple zeros in $D_{2}$ denoted by $\left\{k_{j}\right\}_{n_{0}+1}^{n_{1}}$;

- $\left(s^{T} S^{A}\right)_{11}(k)$ has $n_{2}-n_{1}$ possible simple zeros in $D_{2}$ denoted by $\left\{k_{j}\right\}_{n_{1}+1}^{n_{2}}$;

- $s_{11}(k)$ has $N-n_{2}$ possible simple zeros in $D_{4}$ denoted by $\left\{k_{j}\right\}_{n_{2}+1}^{N}$;

and that none of these zeros coincide. Moreover, we assume that none of these functions have zeros on the boundaries of the $D_{n}$ 's.

We determine the residue conditions at these zeros in the following:

Proposition 2.3. Let $\left\{M_{n}\right\}_{1}^{4}$ be the eigenfunctions defined by (2.14) and assume that the set $\left\{k_{j}\right\}_{1}^{N}$ of singularities are as the above assumption. Then the following residue conditions hold:

$$
\begin{gathered}
\operatorname{Res}_{k=k_{j}}[M]_{1}=\frac{s_{33}\left(k_{j}\right)\left[M\left(k_{j}\right)\right]_{2}-s_{23}\left(k_{j}\right)\left[M\left(k_{j}\right)\right]_{3}}{\dot{s}_{33}\left(k_{j}\right) m_{22}(s)\left(k_{j}\right)} e^{2 \theta\left(k_{j}\right)}, \quad 1 \leq j \leq n_{0}, k_{j} \in D_{1} \\
\operatorname{Res}_{k=k_{j}}[M]_{1}=\frac{S_{21}\left(k_{j}\right) s_{33}\left(k_{j}\right)-S_{31}\left(k_{j}\right) s_{23}\left(k_{j}\right)}{\left(S^{T} s^{A}\right)} e^{2 \theta\left(k_{j}\right) m_{11}\left(k_{j}\right)}\left[M\left(k_{j}\right)\right]_{2} \\
+\frac{S_{31}\left(k_{j}\right) s_{22}\left(k_{j}\right)-S_{21}\left(k_{j}\right) s_{32}\left(k_{j}\right)}{\left(S^{T} s^{A}\right){ }_{33}\left(k_{j}\right) m_{11}\left(k_{j}\right)} e^{2 \theta\left(k_{j}\right)}\left[M\left(k_{j}\right)\right]_{3} \\
n_{0}+1 \leq j \leq n_{1}, k_{j} \in D_{2}, \\
n_{1}+1 \leq j \leq n_{2}, k_{j} \in D_{3}, \\
\operatorname{Res}_{k=k_{j}}[M]_{2}=\frac{m_{33}(s)\left(k_{j}\right) M_{21}(S)\left(k_{j}\right)-m_{23}(s)\left(k_{j}\right) M_{31}(S)\left(k_{j}\right)}{\left(s^{T} S^{A}\right)_{11}\left(k_{j}\right) s_{11}\left(k_{j}\right)} e^{-2 \theta\left(k_{j}\right)}\left[M\left(k_{j}\right)\right]_{1} \\
\operatorname{Res}_{k=k_{j}}[M]_{3}=\frac{m_{32}(s)\left(k_{j}\right) M_{21}(S)\left(k_{j}\right)-m_{22}(s)\left(k_{j}\right) M_{31}(S)\left(k_{j}\right)}{\left(s^{T} S^{A}\right)_{11}\left(k_{j}\right) s_{11}\left(k_{j}\right)} e^{-2 \theta\left(k_{j}\right)}\left[M\left(k_{j}\right)\right]_{1} \\
n_{1}+1 \leq j \leq n_{2}, k_{j} \in D_{3} . \\
\operatorname{Res}_{k=k_{j}}[M]_{2}=\frac{m_{33}(s)\left(k_{j}\right)}{\dot{s}_{11}\left(k_{j}\right) s_{21}\left(k_{j}\right)} e^{-2 \theta\left(k_{j}\right)\left[M\left(k_{j}\right)\right]_{1}, \quad n_{2}+1 \leq j \leq N, k_{j} \in D_{4} .} \\
\operatorname{Res}_{k=k_{j}}[M]_{3}=\frac{m_{32}(s)\left(k_{j}\right)}{\dot{s}_{11}\left(k_{j}\right) s_{21}\left(k_{j}\right)} e^{-2 \theta\left(k_{j}\right)\left[M\left(k_{j}\right)\right]_{1}, \quad} n_{2}+1 \leq j \leq N, k_{j} \in D_{4} .
\end{gathered}
$$

where $\dot{f}=\frac{d f}{d k}$, and $\theta$ is defined by

$$
\theta(x, t, k)=i k x+2 i k^{2} t .
$$


Proof. We will prove (2.32a), (2.32c), the other conditions follow by similar arguments. Equation (2.26) implies the relation

$$
\begin{aligned}
& M_{1}=\mu_{2} e^{\left(i k x+2 i k^{2} t\right) \hat{\Lambda}} S_{1}, \\
& M_{3}=\mu_{2} e^{\left(i k x+2 i k^{2} t\right) \hat{\Lambda}} S_{3},
\end{aligned}
$$

In view of the expressions for $S_{1}$ and $S_{3}$ given in (2.27), the three columns of (2.34a) read:

$$
\begin{gathered}
{\left[M_{1}\right]_{1}=\left[\mu_{2}\right]_{1} \frac{1}{m_{11}(s)},} \\
{\left[M_{1}\right]_{2}=\left[\mu_{2}\right]_{1} e^{-2 \theta} s_{12}+\left[\mu_{2}\right]_{2} s_{22}+\left[\mu_{2}\right]_{3} s_{32},} \\
{\left[M_{1}\right]_{3}=\left[\mu_{2}\right]_{1} e^{-2 \theta} s_{13}+\left[\mu_{2}\right]_{2} s_{23}+\left[\mu_{2}\right]_{3} s_{33} .}
\end{gathered}
$$

while the three columns of (2.34b) read:

$$
\begin{aligned}
{\left[M_{3}\right]_{1}=} & {\left[\mu_{2}\right]_{1} s_{11}+\left[\mu_{2}\right]_{2} s_{21} e^{2 \theta}+\left[\mu_{2}\right]_{3} s_{31} e^{2 \theta} } \\
{\left[M_{3}\right]_{2} } & =\left[\mu_{2}\right]_{1} \frac{m_{33}(s) M_{21}(S)-m_{23}(s) M_{31}(S)}{\left(s^{T} S^{A}\right)_{11}} e^{-2 \theta} \\
& +\left[\mu_{2}\right]_{2} \frac{m_{33}(s) M_{11}(S)-m_{13}(s) M_{31}(S)}{\left(s^{T} S^{A}\right)_{11}} \\
& +\left[\mu_{2}\right]_{3} \frac{m_{23}(s) M_{11}(S)-m_{13}(s) M_{21}(S)}{\left(s^{T} S^{A}\right)_{11}} \\
{\left[M_{3}\right]_{3} } & =\left[\mu_{2}\right]_{1} \frac{m_{32}(s) M_{21}(S)-m_{22}(s) M_{31}(S)}{\left(s^{T} S^{A}\right)_{11}} e^{-2 \theta} \\
& +\left[\mu_{2}\right]_{2} \frac{m_{32}(s) M_{11}(S)-m_{12}(s) M_{31}(S)}{\left(S^{T} S^{A}\right)_{11}} \\
& +\left[\mu_{2}\right]_{3} \frac{m_{22}(s) M_{11}(S)-m_{12}(s) M_{21}(S)}{\left(s^{T} S^{A}\right)_{11}}
\end{aligned}
$$

We first suppose that $k_{j} \in D_{1}$ is a simple zero of $m_{11}(s)(k)$. Solving (2.35b) and (2.35c) for $\left[\mu_{2}\right]_{1}$ and substituting the result in to (2.35a), we find

$$
\left[M_{1}\right]_{1}=\frac{s_{33}\left[M_{1}\right]_{2}-s_{23}\left[M_{1}\right]_{3}}{m_{11}(s) m_{22}(s)} e^{2 \theta}-\frac{\left[\mu_{2}\right]_{2}}{m_{21}(s)} e^{2 \theta} .
$$

Taking the residue of this equation at $k_{j}$, we find the condition (2.32a) in the case when $k_{j} \in D_{1}$.

In order to prove (2.32c), we solve (2.36a) for $\left[\mu_{2}\right]_{1}$, then substituting the result into (2.36b) and (2.36c), we find

$$
\begin{aligned}
& {\left[M_{3}\right]_{2}=\frac{m_{33}(s)}{s_{11}}\left[\mu_{2}\right]_{2}+\frac{m_{23}(s)}{s_{11}}\left[\mu_{2}\right]_{3}+\frac{m_{33}(s) M_{21}(S)-m_{23}(s) M_{31}(S)}{\left(s^{T} \dot{S}^{A}\right)_{11} s_{11}} e^{-2 \theta}\left[M_{3}\right]_{1},} \\
& {\left[M_{3}\right]_{3}=\frac{m_{32}(s)}{s_{11}}\left[\mu_{2}\right]_{2}+\frac{m_{22}(s)}{s_{11}}\left[\mu_{2}\right]_{3}+\frac{m_{32}(s) M_{21}(S)-m_{22}(s) M_{31}(S)}{\left(s^{T} \dot{S}^{A}\right)_{11} s_{11}} e^{-2 \theta}\left[M_{3}\right]_{1} .}
\end{aligned}
$$

Taking the residue of this equation at $k_{j}$, we find the condition (2.32c) in the case when $k_{j} \in D_{3}$. 


\subsection{The global relation}

The spectral functions $S(k)$ and $s(k)$ are not independent but satisfy an important relation. Indeed, it follows from (2.24) that

$$
\mu_{1}(x, t, k) e^{\left(i k x+2 i k^{2} t\right) \hat{\Lambda}} S^{-1}(k) s(k)=\mu_{3}(x, t, k), \quad k \in\left(D_{3} \cup D_{4}, D_{3} \cup D_{4}, D_{1} \cup D_{2}\right) .
$$

Since $\mu_{1}(0, T, k)=\mathbb{I}$, evaluation at $(0, T)$ yields the following global relation:

$$
S^{-1}(k) s(k)=e^{-2 i k^{2} T \hat{\Lambda}} c(T, k), \quad k \in\left(D_{3} \cup D_{4}, D_{3} \cup D_{4}, D_{1} \cup D_{2}\right) .
$$

where $c(T, k)=\mu_{3}(0, T, k)$.

\section{The Riemann-Hilbert problem}

In this section, we state our main result that the solutions $q_{1}(x, t)$ and $q_{2}(x, t)$ of (1.2) can be recovered from a $3 \times 3$ Riemann-Hilbert problem.

Theorem 3.1. Suppose that $q_{1}(x, t)$ and $q_{2}(x, t)$ are a pair of solutions of $(1.2)$ in the half-line domain $\Omega$ with sufficient smoothness and decays as $x \rightarrow \infty$. Then $q_{1}(x, t)$ and $q_{2}(x, t)$ can be reconstructed from the initial value $\left\{q_{10}(x), q_{20}(x)\right\}$ and boundary values $\left\{g_{01}(t), g_{02}(t), g_{11}(t), g_{12}(t)\right\}$ defined as follows,

$$
\begin{aligned}
& q_{10}(x)=q_{1}(x, 0), q_{20}(x)=q_{2}(x, 0), g_{01}(t)=q_{1}(0, t), \\
& g_{02}(t)=q_{2}(0, t), g_{11}(t)=q_{1 x}(0, t), g_{12}(t)=q_{2 x}(0, t) .
\end{aligned}
$$

Use the initial and boundary data to define the jump matrices $J_{m, n}(x, t, k)$ as well as the spectral $s(k)$ and $S(k)$ by equation (2.24). Assume that the possible zeros $\left\{k_{j}\right\}_{1}^{N}$ of the functions $m_{11}(s)(k),\left(S^{T} s^{A}\right)_{11}(k),\left(s^{T} S^{A}\right)_{11}(k)$ and $s_{11}(k)$ are as in assumption 2.3.

Then the solution $\left\{q_{1}(x, t), q_{2}(x, t)\right\}$ is given by

$$
q_{1}(x, t)=2 i \lim _{k \rightarrow \infty}(k M(x, t, k))_{12}, \quad q_{2}(x, t)=2 i \lim _{k \rightarrow \infty}(k M(x, t, k))_{13} .
$$

where $M(x, t, k)$ satisfies the following $3 \times 3$ matrix Riemann-Hilbert problem:

- $M$ is sectionally meromorphic on the Riemann $k$-sphere with jumps across the contours $\bar{D}_{n} \cap \bar{D}_{m}, n, m=1, \cdots, 4$, see Figure 2.

- Across the contours $\bar{D}_{n} \cap \bar{D}_{m}$, M satisfies the jump condition

$$
M_{n}(x, t, k)=M_{m}(x, t, k) J_{m, n}(x, t, k), \quad k \in \bar{D}_{n} \cap \bar{D}_{m}, n, m=1,2,3,4 .
$$

- $M(x, t, k)=\mathbb{I}+O\left(\frac{1}{k}\right), \quad k \rightarrow \infty$.

- The residue condition of $M$ is showed in Proposition 2.3. 
Proof. It only remains to prove (3.2) and this equation follows from the large $k$ asymptotics of the eigenfunctions.

\section{Non-linearizable Boundary Conditions}

As we state in the introduction, the most difficulty of initial-boundary value problems is that some of the boundary values are unkown for a well-posed problem. All boundary values are needed for the definition of $S(k)$, and hence for the formulation of the Riemann-Hilbert problem. Our main result in this section expresses the spectral function $S(k)$ in terms of the prescribed boundary data and the initial data via the solution of a system of nonlinear integral equations.

\subsection{Asymptotics}

An analysis of (2.7) shows that the eigenfunctions $\left\{\mu_{j}\right\}_{1}^{3}$ have the following asymptotics as $k \rightarrow \infty$ :

$$
\begin{aligned}
& \mu_{j}(x, t, k)=\mathbb{I}+\frac{1}{k}\left(\begin{array}{ccc}
\mu_{11}^{(1)} & \mu_{12}^{(1)} & \mu_{13}^{(1)} \\
\mu_{21}^{(1)} & \mu_{22}^{(1)} & \mu_{23}^{(1)} \\
\mu_{31}^{(1)} & \mu_{32}^{(1)} & \mu_{33}^{(1)}
\end{array}\right)+\frac{1}{k^{2}}\left(\begin{array}{lll}
\mu_{11}^{(2)} & \mu_{12}^{(2)} & \mu_{13}^{(2)} \\
\mu_{21}^{(2)} & \mu_{22}^{(2)} & \mu_{23}^{(2)} \\
\mu_{31}^{(2)} & \mu_{32}^{(2)} & \mu_{33}^{(2)}
\end{array}\right)+O\left(\frac{1}{k^{3}}\right) \\
& =\mathbb{I}+\frac{1}{k}\left(\begin{array}{ccc}
\int_{\left(x_{j}, t_{j}\right)}^{(x, t)} \Delta_{11} & \frac{q_{1}}{2 i} & \frac{q_{2}}{2 i} \\
-\frac{\bar{q}_{1}}{2 i} & \int_{\left(x_{j}, t_{j}\right)}^{(x, t)} \Delta_{22}^{(1)} \int_{\left(x_{j}, t_{j}\right)}^{(x, t)} \Delta_{23}^{(1)} \\
-\frac{\bar{q}_{2}}{2 i} & \int_{(x, t)}^{(x, t)} \Delta_{32}^{(1)} \int_{\left(x_{j}, t_{j}\right)}^{(x, t)} \Delta_{33}^{(1)}
\end{array}\right) \\
& +\frac{1}{k^{2}}\left(\begin{array}{ccc}
\int_{\left(x_{j}, t_{j}\right)}^{(x, t)} \eta_{j} & \frac{q_{1} \mu_{22}^{(1)}-q_{2} \mu_{32}^{(1)}-\mu_{12 x}^{(1)}}{2 i} & \frac{q_{1} \mu_{23}^{(1)}-q_{2} \mu_{33}^{(1)}-\mu_{13 x}^{(1)}}{2 i} \\
\frac{\mu_{21 x}^{(1)}-\bar{q}_{1} \mu_{11}^{(1)}}{2 i} & \int_{\left(x_{j}, t_{j}\right)}^{(x, t)} \Delta_{22}^{(2)} & \int_{\left(x_{j}, t_{j}\right)}^{(x, t)} \Delta_{23}^{(2)} \\
\frac{\mu_{31 x}^{(1)}-\bar{q}_{2} \mu_{11}^{(1)}}{2 i} & \int_{\left(x_{j}, t_{j}\right)}^{(x, t)} \Delta_{32}^{(2)} & \int_{\left(x_{j}, t_{j}\right)}^{(x, t)} \Delta_{33}^{(2)}
\end{array}\right)+O\left(\frac{1}{\left.k^{3}\right)}\right) .
\end{aligned}
$$

where

$$
\begin{aligned}
& \Delta_{11}=\frac{i}{2}\left(\left|q_{1}\right|^{2}+\left|q_{2}\right|^{2}\right) d x+\frac{1}{2}\left(q_{1} \bar{q}_{1 x}-\bar{q}_{1} q_{1 x}+q_{2} \bar{q}_{2 x}-\bar{q}_{2} q_{2 x}\right) d t \\
& \Delta_{22}^{(1)}=-\frac{i}{2}\left|q_{1}\right|^{2} d x-\frac{1}{2}\left(\bar{q}_{1 x} q_{1}-\bar{q}_{1} q_{1 x}\right) d t \\
& \Delta_{23}^{(1)}=-\frac{i}{2} \bar{q}_{1} q_{2} d x-\frac{1}{2}\left(\bar{q}_{1 x} q_{2}-\bar{q}_{1} q_{2 x}\right) d t \\
& \Delta_{32}^{(1)}=-\frac{i}{2} \bar{q}_{2} q_{1} d x-\frac{1}{2}\left(\bar{q}_{2 x} q_{1}-\bar{q}_{2} q_{1 x}\right) d t \\
& \Delta_{33}^{(1)}=-\frac{i}{2}\left|q_{2}\right|^{2} d x-\frac{1}{2}\left(\bar{q}_{2 x} q_{2}-\bar{q}_{2} q_{2 x}\right) d t
\end{aligned}
$$


$\Delta_{22}^{(2)}=\left(-\frac{i}{2}\left|q_{1}\right|^{2} \mu_{22}^{(1)}-\frac{i}{2} \bar{q}_{1} q_{2} \mu_{32}^{(1)}+\frac{1}{4} \bar{q}_{1} q_{1 x}\right) d x$

$+\left[-\frac{1}{2}\left(\bar{q}_{1 x} q_{1}-\bar{q}_{1} q_{1 x}\right) \mu_{22}^{(1)}-\frac{1}{2}\left(\bar{q}_{1 x} q_{2}-\bar{q}_{1} q_{2 x}\right) \mu_{32}^{(1)}-\frac{i}{4}\left(\left|q_{1}\right|^{4}+\left|q_{1}\right|^{2}\left|q_{2}\right|^{2}\right)+\frac{i}{4}\left(\bar{q}_{1} q_{1 x x}-\bar{q}_{1 x} q_{1 x}\right)\right] d t$

$\Delta_{23}^{(2)}=\left(-\frac{i}{2}\left|q_{1}\right|^{2} \mu_{23}^{(1)}-\frac{i}{2} \bar{q}_{1} q_{2} \mu_{33}^{(1)}+\frac{1}{4} \bar{q}_{1} q_{2 x}\right) d x$

$+\left[-\frac{1}{2}\left(\bar{q}_{1 x} q_{1}-\bar{q}_{1} q_{1 x}\right) \mu_{23}^{(1)}-\frac{1}{2}\left(\bar{q}_{1 x} q_{2}-\bar{q}_{1} q_{2 x}\right) \mu_{33}^{(1)}-\frac{i}{4}\left(\left|q_{1}\right|^{2} \bar{q}_{1} q_{2}+\bar{q}_{1} q_{2}\left|q_{2}\right|^{2}\right)+\frac{i}{4}\left(\bar{q}_{1} q_{2 x x}-\bar{q}_{1 x} q_{2 x}\right)\right] d t$

$\Delta_{32}^{(2)}=\left(-\frac{i}{2} \bar{q}_{2} q_{1} \mu_{22}^{(1)}-\frac{i}{2}\left|q_{2}\right|^{2} \mu_{32}^{(1)}+\frac{1}{4} \bar{q}_{2} q_{1 x}\right) d x$

$+\left[-\frac{1}{2}\left(\bar{q}_{2 x} q_{1}-\bar{q}_{2} q_{1 x}\right) \mu_{22}^{(1)}-\frac{1}{2}\left(\bar{q}_{2 x} q_{2}-\bar{q}_{2} q_{2 x}\right) \mu_{32}^{(1)}-\frac{i}{4}\left(\left|q_{1}\right|^{2} \bar{q}_{2} q_{1}+\left|q_{2}\right|^{2} \bar{q}_{2} q_{1}\right)+\frac{i}{4}\left(\bar{q}_{2} q_{1 x x}-\bar{q}_{2 x} q_{1 x}\right)\right] d t$

$\Delta_{33}^{(2)}=\left(-\frac{i}{2} \bar{q}_{2} q_{1} \mu_{23}^{(1)}-\frac{i}{2}\left|q_{2}\right|^{2} \mu_{33}^{(1)}+\frac{1}{4} \bar{q}_{2} q_{2 x}\right) d x$

$+\left[-\frac{1}{2}\left(\bar{q}_{2 x} q_{1}-\bar{q}_{2} q_{1 x}\right) \mu_{23}^{(1)}-\frac{1}{2}\left(\bar{q}_{2 x} q_{2}-\bar{q}_{2} q_{2 x}\right) \mu_{33}^{(1)}-\frac{i}{4}\left(\left|q_{1}\right|^{2}\left|q_{2}\right|^{2}+\left|q_{2}\right|^{4}\right)+\frac{i}{4}\left(\bar{q}_{2} q_{2 x x}-\bar{q}_{2 x} q_{2 x}\right)\right] d t$.

We define functions $\left\{\Phi_{i j}(t, k)\right\}_{i, j=1}^{3}$ by:

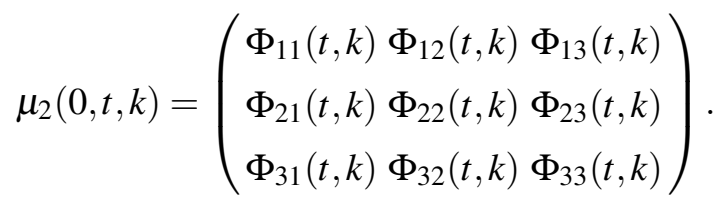

From the global relation (2.39)and replacing $T$ by $t$, we find

$$
\mu_{2}(0, t, k) e^{2 i k^{2} t \hat{\Lambda}} s(k)=c(t, k), \quad k \in\left(D_{3} \cup D_{4}, D_{1} \cup D_{2}, D_{1} \cup D_{2}\right) .
$$

We partition matrix as following,

$$
\mu_{2}(0, t, k)=\left(\begin{array}{cc}
\Phi_{11} & \Phi_{1 j} \\
\Phi_{j 1} & \Phi_{2 \times 2}
\end{array}\right), \quad j=2,3
$$

where $\Phi_{2 \times 2}$ denotes a $2 \times 2$ matrix, $\Phi_{1 j}$ denotes a $1 \times 2$ vector, $\Phi_{j 1}$ denotes a $2 \times 1$ vector. Then, we can write the second column of the global relation, undering the matrix partitioned as (4.5), as

$$
\begin{gathered}
\Phi_{11}(t, k) s_{1 j}(k) s_{2 \times 2}^{-1}(k) e^{-4 i k^{2} t}+\Phi_{1 j}(t, k)=c_{1 j}(t, k), \quad k \in D_{1} \cup D_{2}, \\
\Phi_{j 1}(t, k) s_{1 j}(k) s_{2 \times 2}^{-1}(k) e^{-4 i k^{2} t}+\Phi_{2 \times 2}(t, k)=c_{2 \times 2}(t, k), \quad k \in D_{1} \cup D_{2},
\end{gathered}
$$

The functions $c_{1 j}(t, k), c_{2 \times 2}(t, k)$ are analytic and bounded in $D_{1} \cup D_{2}$ away from the possible zeros of $m_{11}(k)$ and of order $O\left(\frac{1}{k}\right)$ as $k \rightarrow \infty$.

From the asymptotic of $\mu_{j}(x, t, k)$ in (4.1a) we have

$$
\begin{aligned}
\mu_{2}(0, t, k)= & \mathbb{I}+\frac{1}{k}\left(\begin{array}{cc}
\int_{(0,0)}^{(0, t)} \Delta_{11} & \frac{1}{2 i} Q \\
-\frac{1}{2 i} \bar{Q}^{T} & \int_{(0,0)}^{(0, t)} \Delta^{(1)}
\end{array}\right) \\
& +\frac{1}{k^{2}}\left(\begin{array}{cc}
\int_{(0, t)}^{(0, t)} \eta_{2} & -\frac{i}{2} Q \int_{(0,0)}^{(0,0)} \Delta^{(1)}+\frac{1}{4} Q_{x} \\
\frac{i}{2} \bar{Q}^{T} \int_{(0,0)}^{(0, t)} \Delta_{11}+\frac{1}{4} \bar{Q}_{x}^{T} & \int_{(0,0)}^{(0, t)} \Delta^{(2)}
\end{array}\right)+O\left(\frac{1}{k^{3}}\right)
\end{aligned}
$$


where $Q=\left(q_{1}, q_{2}\right), \Delta_{11}$ is defined by first identities of (4.2a), $\eta_{2}$ is defined by (4.2b), $\Delta^{(1)}$ and $\Delta^{(2)}$ are $2 \times 2$ matrices defined as following,

$$
\Delta^{(j)}=\left(\begin{array}{ll}
\Delta_{22}^{(j)} & \Delta_{23}^{(j)} \\
\Delta_{32}^{(j)} & \Delta_{33}^{(j)}
\end{array}\right), \quad j=1,2,
$$

here, $\left\{\Delta_{k l}^{(j)}\right\}_{k, l=2,3}, j=1,2$ are defined as (4.2a) and (4.2c). Also, we have

$$
\begin{gathered}
\Phi_{1 j}(t, k)=\frac{\Phi_{1 j}^{(1)}(t)}{k}+\frac{\Phi_{1 j}^{(2)}(t)}{k^{2}}+O\left(\frac{1}{k^{3}}\right), \quad k \rightarrow \infty, k \in D_{1} \cup D_{2} \\
\Phi_{2 \times 2}(t, k)=\mathbb{I}_{2 \times 2}+\frac{\Phi_{2 \times 2}^{(1)}(t)}{k}+\frac{\Phi_{2 \times 2}^{(2)}(t)}{k^{2}}+O\left(\frac{1}{k^{3}}\right), \quad k \rightarrow \infty, k \in D_{1} \cup D_{2} .
\end{gathered}
$$

where

$$
\begin{aligned}
& \Phi_{1 j}^{(1)}(t)=\frac{1}{2 i} g_{0}(t), \quad \Phi_{1 j}^{(2)}(t)=\frac{1}{4} g_{1}(t)-\frac{i}{2} g_{0} \int_{(0,0)}^{(0, t)} \Delta^{(1)} \\
& \Phi_{2 \times 2}^{(1)}(t)=\int_{(0,0)}^{(0, t)} \Delta^{(1)}, \Phi_{2 \times 2}^{(2)}(t)=\int_{(0,0)}^{(0, t)} \Delta^{(2)}
\end{aligned}
$$

here $g_{0}(t)$ and $g_{1}(t)$ are vector boundary functions defined by the boundary data of (1.3) as $g_{0}(t)=$ $\left(g_{01}(t), g_{02}(t)\right)$ and $g_{1}(t)=\left(g_{11}(t), g_{12}(t)\right)$.

In particular, we find the following expressions for the boudary values:

$$
\begin{gathered}
g_{0}=2 i \Phi_{1 j}^{(1)}(t), \\
g_{1}=2 i g_{0} \Phi_{2 \times 2}^{(1)}(t)+4 \Phi_{1 j}^{(2)}(t),
\end{gathered}
$$

We will also need the asymptotic of $c_{1 j}(t, k)$,

Lemma 4.1. The global relation (4.6) implies that the large $k$ behavior of $c_{1 j}(t, k), c_{2 \times 2}(t, k)$ satisfies

$$
c_{1 j}(t, k)=\frac{\Phi_{1 j}^{(1)}(t)}{k}+\frac{\Phi_{1 j}^{(2)}(t)}{k^{2}}+O\left(\frac{1}{k^{3}}\right), \quad k \rightarrow \infty, k \in D_{1} .
$$

Proof. Let

$$
\mu_{2}(0, t, k)=\left(\begin{array}{ll}
\Phi_{11} & \Phi_{1 j} \\
\Phi_{j 1} & \Phi_{2 \times 2}
\end{array}\right), \quad j=2,3 .
$$

The global relation shows that

$$
\Phi_{11}(t, k) s_{1 j}(k) s_{2 \times 2}^{-1}(k) e^{-4 i k^{2} t}+\Phi_{1 j}(t, k)=c_{1 j}(t, k) .
$$


And from equation

$$
\mu_{t}-2 i k^{2}[\Lambda, \mu]=V_{2} \mu
$$

From the second column of the equation (4.13) we get

$$
\left\{\begin{array}{l}
\Phi_{1 j t}+4 i k^{2} \Phi_{1 j}=2 k Q \Phi_{2 \times 2}-i k Q \bar{Q}^{T} \Phi_{1 j}+i Q_{x} \Phi_{2 \times 2} \\
\Phi_{2 \times 2 t}=2 k \bar{Q} \Phi_{1 j}+i \bar{Q}^{T} Q \Phi_{2 \times 2}-i \bar{Q}_{x}^{T} \Phi_{1 j}
\end{array}\right.
$$

where $Q=\left(q_{1}, q_{2}\right)$ is a $1 \times 2$ vector function.

Suppose

$$
\left(\begin{array}{l}
\Phi_{1 j} \\
\Phi_{2 \times 2}
\end{array}\right)=\left(\alpha_{0}(t)+\frac{\alpha_{1}(t)}{k}+\frac{\alpha_{2}(t)}{k^{2}}+\cdots\right)+\left(\beta_{0}(t)+\frac{\beta_{1}(t)}{k}+\frac{\beta_{2}(t)}{k^{2}}+\cdots\right) e^{-4 i k^{2} t}
$$

where the coefficients $\alpha_{l}(t)$ and $\beta_{l}(t), l \geq 0$, are independent of $k$. To determine these coefficients, we substitute the above equation into equation (4.14) and use the initial conditions

$$
\alpha_{0}(0)+\beta_{0}(0)=\left(0_{1 \times 2}, \mathbb{I}_{2 \times 2}\right)^{T}, \quad \alpha_{1}(0)+\beta_{1}(0)=\left(0_{1 \times 2}, 0_{2 \times 2}\right)^{T} .
$$

Then we get

$$
\begin{aligned}
& \left(\begin{array}{l}
\Phi_{1 j} \\
\Phi_{2 \times 2}
\end{array}\right)=\left(\begin{array}{c}
0_{1 \times 2} \\
\mathbb{I}_{2 \times 2}
\end{array}\right)+\frac{1}{k}\left(\begin{array}{l}
\Phi_{1 j}^{(1)} \\
\Phi_{2 \times 2}^{(1)}
\end{array}\right)+O\left(\frac{1}{k^{2}}\right) \\
& +\left[\frac{1}{k}\left(\begin{array}{l}
\frac{-Q(0)}{2 i} \\
0
\end{array}\right)+O\left(\frac{1}{k^{2}}\right)\right] e^{-4 i k^{2} t}
\end{aligned}
$$

From the first column of the equation (4.13) we get

$$
\left\{\begin{array}{l}
\Phi_{11 t}=2 k Q \Phi_{j 1}-i Q \bar{Q}^{T} \Phi_{11}+i Q_{x} \Phi_{j 1} \\
\Phi_{j 1 t}-4 i k^{2} \Phi_{j 1}=2 k \bar{Q}^{T} \Phi_{11}+i \bar{Q}^{T} Q \Phi_{j 1}-i \bar{Q}_{x}^{T} \Phi_{11}
\end{array}\right.
$$

Suppose

$$
\left(\begin{array}{l}
\Phi_{2 \times 2} \\
\Phi_{3 j}
\end{array}\right)=\left(\xi_{0}(t)+\frac{\xi_{1}(t)}{k}+\frac{\xi_{2}(t)}{k^{2}}+\cdots\right)+\left(v_{0}(t)+\frac{v_{1}(t)}{k}+\frac{v_{2}(t)}{k^{2}}+\cdots\right) e^{8 i k^{3} t}
$$

where the coefficients $\xi_{l}(t)$ and $v_{l}(t), l \geq 0$, are independent of $k$. To determine these coefficients,we substitute the above equation into equation (4.17) and use the initial conditions

$$
\xi_{0}(0)+v_{0}(0)=\left(1,0_{2 \times 1}\right)^{T}
$$


Then we get

$$
\begin{aligned}
\left(\begin{array}{l}
\Phi_{11} \\
\Phi_{j 1}
\end{array}\right)= & \left(\begin{array}{l}
1 \\
0_{2 \times 1}
\end{array}\right)+\frac{1}{k}\left(\begin{array}{l}
\Phi_{11}^{(1)} \\
\Phi_{j 1}^{(1)}
\end{array}\right)+O\left(\frac{1}{k^{2}}\right) \\
& +\left[\frac{1}{k}\left(\begin{array}{l}
0 \\
\frac{\bar{Q}^{T}(0)}{2 i}
\end{array}\right)+O\left(\frac{1}{k^{2}}\right)\right] e^{4 i k^{2} t}
\end{aligned}
$$

So, from the equation (4.12) and the asymptotic of $s_{j 3}(k)$ and $s_{33}(k)$, we get the asymptotic behavior of $c_{j}(t, k)$ as $k \rightarrow \infty$,

$$
c_{j}(t, k)=\frac{\Phi_{j 3}^{(1)}}{k}+\frac{\Phi_{j 3}^{(2)}}{k^{2}}+\frac{\Phi_{j 3}^{(3)}}{k^{3}}+\cdots
$$

\subsection{The Dirichlet and Neumann problems}

We can now derive effective characterizations of spectral function $S(k)$ for the Dirichlet $\left(g_{0}\right.$ prescribed), the Neumann ( $g_{1}$ prescribed) problems.

Define functions as

$$
\Omega(t, k)=\Phi_{1 j}(t, k)-\Phi_{1 j}(t,-k), \quad \hat{\Omega}(t, k)=\Phi_{2 \times 2}(t, k)-\Phi_{2 \times 2}(t,-k),
$$

and

$$
\omega(t, k)=\Phi_{1 j}(t, k)+\Phi_{1 j}(t,-k), \quad \tilde{\omega}(t, k)=\Phi_{2 \times 2}(t, k)+\Phi_{2 \times 2}(t,-k)
$$

Theorem 4.1. Let $T<\infty$. Let $q_{0}(x)=\left(q_{10}(x), q_{20}(x)\right), x \geq 0$, be a vector function of Schwartz class.

For the Dirichlet problem it is assumed that the function $g_{0}(t), 0 \leq t<T$, has sufficient smoothness and is compatible with $q_{0}(x)$ at $x=t=0$.

For the Neumann problem it is assumed that the function $g_{1}(t), 0 \leq t<T$, has sufficient smoothness and is compatible with $q_{0}(x)$ at $x=t=0$.

Suppose that $m_{11}(k)$ has a finite number of simple zeros in $D_{1}$.

Then the spectral function $S(k)$ is given by

$$
S(k)=\left(\begin{array}{ccc}
A(k) & e^{-4 i k^{2} T} B(k) & e^{-4 i k^{2} T} C(k) \\
e^{4 i k^{2} T} D(k) & E(k) & F(k) \\
e^{4 i k^{2} T} G(k) & H(k) & I(k)
\end{array}\right)
$$


where

$$
\begin{aligned}
& A(k)=\Phi_{22}(k) \Phi_{33}(k)-\Phi_{23}(k) \Phi_{32}(k) B(k)=\Phi_{13}(k) \Phi_{22}(k)-\Phi_{12}(k) \Phi_{33}(k) \\
& C(k)=\Phi_{12}(k) \Phi_{23}(k)-\Phi_{13}(k) \Phi_{22}(k) D(k)=\Phi_{23}(k) \Phi_{31}(k)-\Phi_{21}(k) \Phi_{33}(k) \\
& E(k)=\Phi_{11}(k) \Phi_{33}(k)-\Phi_{13}(k) \Phi_{31}(k) F(k)=\Phi_{21}(k) \Phi_{13}(k)-\Phi_{11}(k) \Phi_{23}(k) \\
& G(k)=\Phi_{21}(k) \Phi_{32}(k)-\Phi_{22}(k) \Phi_{31}(k) H(k)=\Phi_{12}(k) \Phi_{31}(k)-\Phi_{11}(k) \Phi_{32}(k) \\
& I(k)=\Phi_{11}(k) \Phi_{22}(k)-\Phi_{12}(k) \Phi_{21}(k)
\end{aligned}
$$

and the complex-value functions $\left\{\Phi_{l 3}(t, k)\right\}_{l=1}^{3}$ satisfy the following system of integral equations:

$$
\begin{gathered}
\Phi_{13}(t, k)=\int_{0}^{t} e^{4 i k^{2}\left(t-t^{\prime}\right)}\left[-i\left(\left|g_{01}\right|^{2}+\left|g_{02}\right|^{2}\right) \Phi_{13}\right. \\
\left.+\left(2 k g_{01}+i g_{11}\right) \Phi_{23}+\left(2 k g_{02}+i g_{12}\right) \Phi_{33}\right]\left(t^{\prime}, k\right) d t^{\prime} \\
\Phi_{23}(t, k)=\int_{0}^{t}\left[\left(2 k \bar{g}_{01}-i \bar{g}_{11}\right) \Phi_{13}+i\left|g_{01}\right|^{2} \Phi_{23}+i \bar{g}_{01} g_{02} \Phi_{33}\right]\left(t^{\prime}, k\right) d t^{\prime} \\
\Phi_{33}(t, k)=1+\int_{0}^{t}\left[\left(2 k \bar{g}_{02}-i \bar{g}_{12}\right) \Phi_{13}+i g_{01} \bar{g}_{02} \Phi_{23}+i\left|g_{02}\right|^{2} \Phi_{33}\right]\left(t^{\prime}, k\right) d t^{\prime}
\end{gathered}
$$

and $\left\{\Phi_{l 1}(t, k)\right\}_{l=1}^{3},\left\{\Phi_{l 2}(t, k)\right\}_{l=1}^{3}$ satisfy the following system of integral equations:

$$
\begin{aligned}
& \Phi_{11}(t, k)=1+\int_{0}^{t}\left[-i\left(\left|g_{01}\right|^{2}+\left|g_{02}\right|^{2}\right) \Phi_{11}\right. \\
& \left.+\left(2 k g_{01}+i g_{11}\right) \Phi_{21}+\left(2 k g_{02}+i g_{12}\right) \Phi_{31}\right]\left(t^{\prime}, k\right) d t^{\prime} \\
& \Phi_{21}(t, k)=\int_{0}^{t} e^{-4 i k^{2}\left(t-t^{\prime}\right)}\left[\left(2 k \bar{g}_{01}-i \bar{g}_{11}\right) \Phi_{11}+i\left|g_{01}\right|^{2} \Phi_{21}+i \bar{g}_{01} g_{02} \Phi_{31}\right]\left(t^{\prime}, k\right) d t^{\prime} \\
& \Phi_{31}(t, k)=\int_{0}^{t} e^{-4 i k^{2}\left(t-t^{\prime}\right)}\left[\left(2 k \bar{g}_{02}-i \bar{g}_{12}\right) \Phi_{11}+i g_{01} \bar{g}_{02} \Phi_{21}+i\left|g_{02}\right|^{2} \Phi_{31}\right]\left(t^{\prime}, k\right) d t^{\prime} \\
& \Phi_{12}(t, k)=\int_{0}^{t} e^{4 i k^{2}\left(t-t^{\prime}\right)}\left[-i\left(\left|g_{01}\right|^{2}+\left|g_{02}\right|^{2}\right) \Phi_{12}\right. \\
& \left.+\left(2 k g_{01}+i g_{11}\right) \Phi_{22}+\left(2 k g_{02}+i g_{12}\right) \Phi_{32}\right]\left(t^{\prime}, k\right) d t^{\prime} \\
& \Phi_{22}(t, k)=1+\int_{0}^{t}\left[\left(2 k \bar{g}_{01}-i \bar{g}_{11}\right) \Phi_{12}+i\left|g_{01}\right|^{2} \Phi_{22}+i \bar{g}_{01} g_{02} \Phi_{32}\right]\left(t^{\prime}, k\right) d t^{\prime} \\
& \Phi_{32}(t, k)=\int_{0}^{t}\left[\left(2 k \bar{g}_{02}-i \bar{g}_{12}\right) \Phi_{12}+i g_{01} \bar{g}_{02} \Phi_{22}+i\left|g_{02}\right|^{2} \Phi_{32}\right]\left(t^{\prime}, k\right) d t^{\prime}
\end{aligned}
$$

(i) For the Dirichlet problem, the unknown Neumann boundary value $g_{1}(t)$ is given by 


$$
\begin{aligned}
g_{1}(t)= & \frac{2}{\pi i} \int_{\partial D_{3}}\left(k \Omega(t, k)+i g_{0}(t)\right) d k+\frac{2 g_{0}(t)}{\pi} \int_{\partial D_{3}} \hat{\Omega}(t, k) d k \\
& -\frac{4}{\pi i} \int_{\partial D_{3}} k e^{-4 i k^{2} t} \Phi_{11}(-k) s_{1 j}(-k) s_{2 \times 2}^{-1}(-k) d k \\
& +8 \sum_{k_{j} \in D_{1}} k_{j} e^{-4 i k_{j}^{2} t} \operatorname{Res}\left(\Phi_{11} s_{1 j} s_{2 \times 2}^{-1}\right)\left(k_{j}\right) .
\end{aligned}
$$

(ii) For the Neumann problem, the unknown boundary values $g_{0}(t)$ is given by

$$
\begin{aligned}
g_{0}(t)= & \frac{1}{\pi} \int_{\partial D_{3}} \omega(t, k) d k+\frac{2}{\pi} \int_{\partial D_{3}} e^{-4 i k^{2} t} \Phi_{11}(-k) s_{1 j}(-k) s_{2 \times 2}^{-1}(-k) d k \\
& +4 i \sum_{k_{j} \in D_{1}} e^{-4 i k_{j}^{2} t} \operatorname{Res}\left(\Phi_{11} s_{1 j} s_{2 \times 2}^{-1}\right)\left(k_{j}\right) .
\end{aligned}
$$

Proof. The representations (4.22) follow from the relation $S(k)=e^{4 i k^{2} T} \mu_{2}^{A}(0, T, k)^{T}$. And the system (4.23) is the direct result of the Volteral integral equations of $\mu_{2}(0, t, k)$.

(i) In order to derive (4.26) we note that equation (4.10b) expresses $g_{1}$ in terms of $\Phi_{2 \times 2}^{(1)}$ and $\Phi_{1 j}^{(2)}$. Furthermore, equation (4.9) and Cauchy theorem imply

$$
-\frac{\pi i}{2} \Phi_{2 \times 2}^{(1)}(t)=\int_{\partial D_{2}}\left[\Phi_{2 \times 2}(t, k)-\mathbb{I}_{2 \times 2}\right] d k=\int_{\partial D_{4}}\left[\Phi_{2 \times 2}(t, k)-\mathbb{I}_{2 \times 2}\right] d k
$$

and

$$
-\frac{\pi i}{2} \Phi_{1 j}^{(2)}(t)=\int_{\partial D_{2}}\left[k \Phi_{1 j}(t, k)-\frac{g_{0}(t)}{2 i}\right] d k=\int_{\partial D_{4}}\left[k \Phi_{1 j}(t, k)-\frac{g_{0}(t)}{2 i}\right] d k
$$

Thus,

$$
\begin{aligned}
& i \pi \Phi_{2 \times 2}^{(1)}(t)=-\left(\int_{\partial D_{2}}+\int_{\partial D_{4}}\right)\left[\Phi_{2 \times 2}(t, k)-\mathbb{I}_{2 \times 2}\right] d k \\
& =\left(\int_{\partial D_{1}}+\int_{\partial D_{3}}\right)\left[\Phi_{2 \times 2}(t, k)-\mathbb{I}_{2 \times 2}\right] d k \\
& =\int_{\partial D_{3}}\left[\Phi_{2 \times 2}(t, k)-\mathbb{I}_{2 \times 2}\right] d k-\int_{\partial D_{3}}\left[\Phi_{2 \times 2}(t,-k)-\mathbb{I}_{2 \times 2}\right] d k \\
& =\int_{\partial D_{3}}\left(\Phi_{2 \times 2}(t, k)-\Phi_{2 \times 2}(t,-k)\right) d k .
\end{aligned}
$$

Similarly,

$$
\begin{aligned}
& i \pi \Phi_{1 j}^{(2)}(t)=\left(\int_{\partial D_{3}}+\int_{\partial D_{1}}\right)\left[k \Phi_{1 j}(t, k)-\frac{g_{0}(t)}{2 i}\right] d k \\
& =\left(\int_{\partial D_{3}}-\int_{\partial D_{1}}\right)\left[k \Phi_{1 j}(t,-k)-\frac{g_{0}(t)}{2 i}\right] d k+I(t) \\
& =\int_{\partial D_{3}}\left[k\left(\Phi_{1 j}(t, k)-\Phi_{1 j}(t,-k)\right)+i g_{0}(t)\right] d k+I(t) .
\end{aligned}
$$

where $I(t)$ is defined by

$$
I(t)=2 \int_{\partial D_{1}}\left[k \Phi_{1 j}(t, k)-\frac{g_{0}(t)}{2 i}\right] d k
$$

The last step involves using the global relation to compute $I(t)$

$$
I(t)=\int_{\partial D_{1}}\left[k\left(\mu_{3,1 j} s_{2 \times 2}^{-1}-\Phi_{11} s_{1 j} e^{-4 i k^{2} t} s_{2 \times 2}^{-1}\right)-\frac{g_{0}(t)}{2 i}\right] d k
$$


Using the asymptotic (4.11) and Cauchy theorem to compute the first term on the right-hand side of equation (4.30), we find

$$
\begin{aligned}
& I(t)=-i \pi \Phi_{1 j}^{(2)}-2 \int_{p t D_{3}} k \Phi_{11}(-k) s_{1 j}(-k) s_{2 \times 2}^{-1}(-k) e^{-4 i k^{2} t} d k \\
& +4 \pi i \sum_{k_{j} \in D_{1}} k_{j} e^{-4 i k_{j}^{2} t} \operatorname{Res}\left(\Phi_{11} s_{1 j} s_{2 \times 2}^{-1}\right)\left(k_{j}\right) .
\end{aligned}
$$

Equations (4.29) and (4.31) imply

$$
\begin{aligned}
& \Phi_{1 j}^{(2)}(t)=\frac{1}{2 \pi i} \int_{\partial D_{3}}\left[k\left(\Phi_{1 j}(t, k)-\Phi_{1 j}(t,-k)\right)+i g_{0}(t)\right] d k \\
& -\frac{1}{\pi i} \int_{p t D_{3}} k \Phi_{11}(-k) s_{1 j}(-k) s_{2 \times 2}^{-1}(-k) e^{-4 i k^{2} t} d k \\
& +2 \sum_{k_{j} \in D_{1}} k_{j} e^{-4 i k_{j}^{2} t} \operatorname{Res}\left(\Phi_{11} s_{1 j} s_{2 \times 2}^{-1}\right)\left(k_{j}\right) .
\end{aligned}
$$

This equation together with (4.10b) and (4.28) yields (4.26).

(ii) In order to derive the representations (4.27) relevant for the Neumann problem, we note that equation (4.10a) expresses $g_{0}$ in terms of $\Phi_{1 j}^{(1)}$. Furthermore, equation (4.9a) and Cauchy's theorem imply

$$
-\frac{\pi i}{2} \Phi_{1 j}^{(1)}(t)=\int_{\partial D_{2}} \Phi_{1 j}(t, k) d k=\int_{\partial D_{4}} \Phi_{1 j}(t, k) d k
$$

Thus,

$$
\begin{aligned}
& i \pi \Phi_{1 j}^{(1)}(t)=\left(\int_{\partial D_{3}}+\int_{\partial D_{1}}\right) \Phi_{1 j}(t, k) d k \\
& =\left(\int_{\partial D_{3}}-\int_{\partial D_{1}}\right) \Phi_{1 j}(t, k) d k+2 \int_{\partial D_{1}} \Phi_{1 j}(t, k) d k \\
& =\int_{\partial D_{3}}\left(\Phi_{1 j}(t, k)+\Phi_{1 j}(t,-k)\right) d k+2 \int_{\partial D_{1}} \Phi_{1 j}(t, k) d k,
\end{aligned}
$$

and using the global relation, we have

$$
\begin{aligned}
& 2 \int_{\partial D_{1}} \Phi_{1 j}(t, k) d k=2 \int_{\partial D_{1}}\left(\mu_{3,1 j} s_{2 \times 2}^{-1}-\Phi_{11} s_{1 j} s_{2 \times 2}^{-1} e^{-4 i k^{2} t}\right) d k \\
& =-i \pi \Phi_{1 j}^{(1)}(t)+2 \int_{\partial D_{3}} \Phi_{11}(-k) s_{1 j}(-k) s_{2 \times 2}^{-1}(-k) e^{-4 i k^{2} t} d k \\
& +4 \pi i \sum_{k_{j} \in D_{1}} e^{-4 i k_{j}^{2} t} \operatorname{Res}\left(\Phi_{11} s_{1 j} s_{2 \times 2}^{-1}\right)\left(k_{j}\right)
\end{aligned}
$$

Equations (4.10a), (4.33) and (4.34) yields (4.27).

\subsection{Effective characterizations}

Substituting into the system (4.23) the expressions

$$
\begin{gathered}
\Phi_{i j}=\Phi_{i j, 0}+\varepsilon \Phi_{i j, 1}+\varepsilon^{2} \Phi_{i j, 2}+\cdots, \quad i, j=1,2,3 . \\
g_{01}=\varepsilon g_{01}^{(1)}+\varepsilon^{2} g_{01}^{(2)}+\cdots, \quad g_{02}=\varepsilon g_{02}^{(1)}+\varepsilon^{2} g_{02}^{(2)}+\cdots,
\end{gathered}
$$




$$
g_{11}=\varepsilon g_{11}^{(1)}+\varepsilon^{2} g_{11}^{(2)}+\cdots, \quad g_{12}=\varepsilon g_{12}^{(1)}+\varepsilon^{2} g_{12}^{(2)}+\cdots,
$$

where $\varepsilon>0$ is a small parameter, we find that the terms of $O(1)$ give

$$
O(1):\left\{\begin{array}{l}
\Phi_{13,0}=0 \Phi_{23,0}=0 \Phi_{33,0}=1, \\
\Phi_{11,0}=1 \Phi_{21,0}=0 \Phi_{31,0}=0, \\
\Phi_{12,0}=0 \Phi_{22,0}=1 \Phi_{32,0}=0 .
\end{array}\right.
$$

Moreover, the terms of $O(\varepsilon)$ give

$$
O(\varepsilon):\left\{\begin{array}{l}
\Phi_{33,1}=0 \quad \Phi_{23,1}=0, \\
\Phi_{13,1}(t, k)=\int_{0}^{t} e^{4 i k^{2}\left(t-t^{\prime}\right)}\left(2 k g_{02}^{(1)}+i g_{12}^{(1)}\right)\left(t^{\prime}\right) d t^{\prime}, \\
\Phi_{11,1}=0, \\
\Phi_{21,1}=\int_{0}^{t} e^{-4 i k^{2}\left(t-t^{\prime}\right)}\left(2 k \bar{g}_{01}^{(1)}-i \bar{g}_{11}^{(1)}\right)\left(t^{\prime}\right) d t^{\prime}, \\
\Phi_{31,1}=\int_{0}^{t} e^{-4 i k^{2}\left(t-t^{\prime}\right)}\left(2 k \bar{g}_{02}^{(1)}-i \bar{g}_{12}^{(1)}\right)\left(t^{\prime}\right) d t^{\prime}, \\
\Phi_{12,1}=\int_{0}^{t} e^{4 i k^{2}\left(t-t^{\prime}\right)}\left(2 k g_{01}^{(1)}+i g_{11}^{(1)}\right)\left(t^{\prime}\right) d t^{\prime}, \\
\Phi_{22,1}=0, \quad \Phi_{32,1}=0 .
\end{array}\right.
$$

and the terms of $O\left(\varepsilon^{2}\right)$ give

$$
O\left(\varepsilon^{2}\right):\left\{\begin{array}{l}
\Phi_{13,2}=\int_{0}^{t} e^{4 i k^{2}\left(t-t^{\prime}\right)}\left(2 k g_{02}^{(2)}+i g_{12}^{(2)}\right)\left(t^{\prime}\right) d t^{\prime}, \\
\Phi_{23,2}=\int_{0}^{t}\left[\left(2 k \bar{g}_{01}^{(1)}-i \bar{g}_{11}^{(1)}\right)\left(t^{\prime}\right) \Phi_{13,1}\left(t^{\prime}, k\right)+i \bar{g}_{01}^{(1)}\left(t^{\prime}\right) g_{02}^{(1)}\left(t^{\prime}\right)\right] d t^{\prime}, \\
\Phi_{33,2}=\int_{0}^{t}\left[\left(2 k \bar{g}_{02}^{(1)}-i \bar{g}_{12}^{(1)}\right)\left(t^{\prime}\right) \Phi_{13,1}\left(t^{\prime}, k\right)+i\left|g_{02}^{(1)}\left(t^{\prime}\right)\right|^{2}\right] d t^{\prime}, \\
\Phi_{11,2}=\int_{0}^{t}\left[-i\left(\left|g_{01}^{(2)}\right|^{2}+\left|g_{02}^{(2)}\right|^{2}\right)\left(t^{\prime}\right)+\left(2 k g_{01}^{(1)}+i g_{11}^{(1)}\right)\left(t^{\prime}\right) \Phi_{21,1}\left(t^{\prime}, k\right)\right. \\
\left.+\left(2 k g_{02}^{(1)}+i g_{12}^{(1)}\right)\left(t^{\prime}\right) \Phi_{31,1}\left(t^{\prime}, k\right)\right] d t^{\prime}, \\
\Phi_{21,2}=\int_{0}^{t} e^{-4 i k^{2}\left(t-t^{\prime}\right)}\left(2 k \bar{g}_{01}^{(2)}-i \bar{g}_{11}^{(2)}\right)\left(t^{\prime}\right) d t^{\prime}, \\
\Phi_{31,2}=\int_{0}^{t} e^{-4 i k^{2}\left(t-t^{\prime}\right)}\left(2 k \bar{g}_{02}^{(2)}-i \bar{g}_{12}^{(2)}\right)\left(t^{\prime}\right) d t^{\prime}, \\
\Phi_{12,2}=\int_{0}^{t} e^{4 i k^{2}\left(t-t^{\prime}\right)}\left(2 k g_{01}^{(2)}+i g_{11}^{(2)}\right)\left(t^{\prime}\right) d t^{\prime}, \\
\Phi_{22,2}=\int_{0}^{t}\left[\left(2 k \bar{g}_{01}^{(1)}-i \bar{g}_{11}^{(1)}\right)\left(t^{\prime}\right) \Phi_{12,1}\left(t^{\prime}, k\right)+i\left|g_{01}^{(1)}\left(t^{\prime}\right)\right|^{2}\right] d t^{\prime}, \\
\Phi_{32,2}=\int_{0}^{t}\left[\left(2 k \bar{g}_{02}^{(1)}-i \bar{g}_{12}^{(1)}\right)\left(t^{\prime}\right) \Phi_{12,1}\left(t^{\prime}, k\right)+i g_{01}^{(1)}\left(t^{\prime}\right) \bar{g}_{02}^{(1)}\left(t^{\prime}\right)\right] d t^{\prime} .
\end{array}\right.
$$

On the other hand, expanding (4.26) and assuming for simplicity that $m_{11}(k)$ has no zeros, we find

$$
g_{1}^{(1)}(t)=\frac{1}{\pi i} \int_{\partial D_{3}}\left(k \Omega^{(1)}(t, k)+i g_{0}^{(1)}\right) d k-\frac{4}{\pi i} \int_{\partial D_{3}} k e^{-4 i k^{2} t} s_{1 j}^{(1)}(-k) s_{2 \times 2}^{(1)-1}(-k) d k
$$

where $\Omega=\varepsilon \Omega^{(1)}+\varepsilon^{2} \Omega^{(2)}+O\left(\varepsilon^{3}\right)$ and $s_{1 j} s_{2 \times 2}^{-1}=\varepsilon s_{1 j}^{(1)} s_{2 \times 2}^{(1)-1}+O\left(\varepsilon^{2}\right)$. 
We also find that

$$
\Omega^{(1)}=4 k \int_{0}^{t} e^{4 i k^{2}\left(t-t^{\prime}\right)} g_{01}^{(1)}\left(t^{\prime}\right) d t^{\prime} .
$$

The Dirichlet problem can now be solved perturbatively as follows: assuming for simplicity that $m_{11}(k)$ has no zeros and given $g_{01}^{(1)}$ and $g_{02}^{(1)}$, we can use equation (4.40) to determine $\Omega^{(1)}$. We can then compute $g_{1}^{(1)}$ from (4.39) and then $\Phi_{1 j, 1}, j=2,3$ from (4.37). In the same way we can determine $\Omega^{(2)}$ and $\hat{\Omega}^{(2)}$ by $g_{0}^{(2)}$, then compute $g_{1}^{(2)}$. And these arguments can be extended to the higher order and also can be extended to the systems (4.24a), (4.25a) and (4.23a), thus yields a constructive scheme for computing $S(k)$ to all orders.

Similarly, these arguments also can be used to the Neumann problem. That is to say, in all cases, the system can be solved perturbatively to all orders.

\section{Acknowledgments}

This work of $\mathrm{Xu}$ was supported by National Science Foundation of China under project NO.11501365, Shanghai Sailing Program supported by Science and Technology Commission of Shanghai Municipality under Grant NO.15YF1408100, Shanghai youth teacher assistance program NO.ZZslg15056 and the Hujiang Foundation of China (B14005).

\section{References}

[1] A. Boutet de Monvel,A.S.Fokas and D.Shepelsky, The mKDV equation on the half-line, J. Inst. Math. Jussieu.3(2004), 139-164.

[2] A. S. Fokas, A unified transform method for solving linear and certain nonlinear PDEs, Proc. R. Soc. Lond. A 453(1997), 1411-1443.

[3] A. S. Fokas, Integrable nonlinear evolution equations on the half-line, Commun. Math. Phys. 230(2002), 1-39.

[4] A.S. Fokas, A Unified Approach to Boundary Value Problems, in: CBMS-NSF Regional Conference Series in Applied Mathematics, SIAM, 2008.

[5] A. S. Fokas, A. R. Its and L. Y. Sung, The nonlinear Schrödinger equation on the half-line, Nonlinearity. 18(2005), 1771-1822.

[6] A. S. Fokas and J. Lenells, The unified method: I.nonlinearizable problem on the half-line, J. Phys. A: Math. Theor. 45(2012) 195201;

[7] C.S. Gardner, J.M. Greene, M.D. Kruskal, and R.M. Miura, Method for the solving for the Korteweg-de Veries equation, Phys. Rev. Lett., 19(1967), 1095-1097.

[8] X. Geng, H. Liu and J. Zhu, Initial-boundary value problems for the coupled nonlinear Schrödinger equation on the half-line, Stud. Appl. Math., 135(2015), 310-346.

[9] P. D. Lax, Integrals of nonlinear equations of evolution and solitary waves, Comm. Pure. Appl. Math.21(1968), 467-490. 
[10] J. Lenells, Initial-boundary value problems for integrable evolution equations with $3 \times 3$ Lax pairs, Physica D 241(2012) 857-875.

[11] J. Lenells, The Degasperis-Procesi equation on the half-line, Nonlinear Analysis 76(2013) 122-139.

[12] J. Lenells and A. S. Forkas, The unified method: II. NLS on the half-line t-periodic boundary conditions, J. Phys. A: Math. Theor. 45(2012) 195202;

[13] J. Lenells and A. S. Forkas, The unified method: III. Nonlinearizable problem on the interval, J. Phys. A: Math. Theor. 45(2012) 195203;

[14] S.V. Manakov, On the theory of two-dimensional stationary self-focusing of electromagenic waves, Sov. Phys. JETP, 38 (1976) 258-254.

[15] J. Xu and E. Fan, The unified transform method for the Sasa-Satsuma equation on the half-line, Proc. R. Soc. A. 469(2013) 20130068.

[16] J. Xu and E. Fan, The three wave equation on the half-line, Physics Letter A, 378(2014) 26-33.

[17] J. Xu and E. Fan, Initial-boundary value problem for integrable nonlinear evolution equations with $3 \times 3$ Lax pairs on the interval, arXiv:1509.02617, to appear in Stud. Appl. Math.. 